\title{
Successful treatment of influenza A encephalopathy
}

Hong Kong Med J 2020;26:154

https://doi.org/10.12809/hkmj208417

To the Editor-Severe complications occur every year in children with influenza, including acute necrotising encephalopathy. ${ }^{1}$ We have previously reported local data on influenza-related childhood mortality and morbidity. ${ }^{2,3}$

In January 2020, in the midst of the COVID19 epidemic and annual influenza and avian influenza seasons, we successfully managed a boy who presented with high fever, four episodes of seizures, and fluctuating consciousness level. Nasopharyngeal aspirate test confirmed influenza A H1. The patient was treated with anticonvulsants and oseltamivir. Cerebral oedema was quickly relieved by hypertonic saline and mannitol infusion. Electroencephalogram showed non-specific slowwave activities. The patient's complete blood counts, C-reactive protein, liver function, and renal function results were within normal limits. The patient became stable after 48 hours of paediatric intensive care. His mother also reported fever and coughing with yellow sputum production but insisted visitation to the intensive care unit despite escalated isolation measures during the COVID-19 epidemic. Neither the mother nor the child had received the seasonal influenza vaccination.

Severe influenza complications occur annually, primarily in unvaccinated children, and caused one to three deaths among Hong Kong children. ${ }^{2}$ We have sufficient Hong Kong literature to reassure members of the public that influenza complications are uncommon in our city, and that influenza vaccination is recommended for prophylaxis. Despite potential serious sequelae, influenza encephalopathy can be successfully treated if prompt critical care management is instituted. ${ }^{4}$

\section{Author contributions}

The author drafted the manuscript, had full access to the data, contributed to the study, approved the final version for publication, and takes responsibility for its accuracy and integrity.

\section{Conflicts of interest}

The author disclosed no conflicts of interest.

\section{Ethics approval}

The patient was treated in accordance with the Declaration of Helsinki. Patient consent was obtained for all investigations and procedures.

\section{KL Hon *, MB, BS, MD}

Department of Paediatrics and Adolescent Medicine, The Hong Kong Children's Hospital, Kowloon Bay, Hong Kong

*Corresponding author: ehon@hotmail.com

\section{References}

1. Lam KF, Wu SW. Two cases of acute necrotising encephalopathy: same disease, different outcomes. HK J Paediatr (New Series) 2019;25:85-9.

2. Hon KL, Tang JW. Low mortality and severe complications despite high influenza burden among Hong Kong children. Hong Kong Med J 2019;25:497-8.

3. Hon KL, Leung TF, Cheung KL, Ng PC, Chan PK. Influenza and parainfluenza associated pediatric ICU morbidity. Indian J Pediatr 2010;77:1097-101.

4. Hon KL, Tsang YC, Chan LC, et al. Outcome of encephalitis in pediatric intensive care unit. Indian J Pediatr 2016;83:1098-103. 